\title{
IAMJ
}

INTERNATIONAL

AYURVEDIC

MEDICAL JOURNAL

Review Article

ISSN: 2320-5091

Impact Factor: 6.719

\section{MIRACLE AYURVEDIC HERB - ASHWAGANDHA (WITHANIA SOMNIFERA DUNAL)}

\section{$\underline{\text { Bargale Sushant Sukumar }}$}

Ph. D Scholar and Assistant Professor, Department of Swasthavritta, Sri Dharmasthala Manjunatheshwara College of Ayurveda and Hospital, Hassan- 573201, Karnataka, India

Corresponding Author: dr.sushant99@gmail.com

\section{https://doi.org/10.46607/iamj2909082021}

(Published Online: August 2021)

Open Access

(C) International Ayurvedic Medical Journal, India 2021

Article Received: 18/07//2021 - Peer Reviewed: 21/07/2021 - Accepted for Publication: 22/07/2021

\section{Check for updates}

\begin{abstract}
Ayurveda is successfully implemented as a primary healthcare system in India, and it is flourishing in the promotion of health around the world. Ayurveda aims to create a society happily, healthy, and peaceful. In general, the simple regimes mentioned in Ayurveda texts are of immense use faced by the present world today regarding health promotion. Ashwagandha (Withania Somnifera) is usually referred to as 'Indian winter cherry' or 'Indian Ginseng'. It is a shrub grown in India and North America whose roots have been used by Ayurvedic practitioners for thousands of years. Ashwagandha is one of Ayurveda's most essential herbs, used as Rasayana for its wide range of health benefits for centuries. Chemicals constitute of Ashwagandha (Withania Somnifera)- The chemistry of Ashwagandha (Withania Somnifera) has been widely studied and numerous categories of chemical constituents have been described, extracted, and isolated, such as steroidal lactones, alkaloids, flavonoids, tannin, etc. More than 12 alkaloids, 40 withanolides and several sitoindosides have currently been isolated from the aerial parts, roots, and berries of the Withania species and have been recorded. The principal biochemical element of Ashwagandha (WS) root is withanolides, which are steroidal alkaloids and steroidal lactones. The pharmacological activity of Ashwagandha- It has been shown to have pharmacological importance as an Adaptogen, Antibiotic, abortifacient, aphrodisiac, Astringent, Anti-inflammatory, deobstructive, diuretic, narcotic, sedative, and tonic through centuries of Ayurvedic medicinal practice using Ashwagandha (Withania Somnifera). Along with these Ashwagandha acts as Anti-stress,
\end{abstract}


Anticarcinogenic activity, Anti-inflammatory activity, Anti-aging activity, Cardioprotective activity, hypothyroid activity and Immunomodulatory activity.

Keywords: Ashwagandha, Withania Somnifera, Alkaloids, Withanoids, sitoindosides

\section{INTRODUCTION}

In traditional Indian systems of medicine, Withania Somnifera Dunal (Solanaceae), also known as Ashwagandha or winter cherry, is one of the most important plants. It is a small evergreen shrub with a height of approximately four to five feet. In India, it is cultivated in the Madhya Pradesh, Uttar Pradesh, Punjab, Gujarat, and Rajasthan states on a commercial scale. In Ayurveda, Unani and Siddha, this plant is used in more than 200 formulations. One of the prime drugs of Ashwagandha is Medica Ayurveda Material. It was included in Balya and Brimhanagana by Acharya Charaka. Balya, Vrishya and Rasayana are attributed to the Properties and recommended as a substitute for Kakoli and Kshirakakoli. ${ }^{1}$ The name somnifera means 'sleep inducing' in Latin, suggesting that sedating properties are attributed to it, but it has also been attributed to it. Used as an adaptogen and used for sexual vitality. Ashwagandha is referred to by some herbalists as Indian ginseng, as it is used in Ayurvedic medicine in a way like that used in traditional Chinese medicine. The Decoction of the roots is used for colds and chills in an ethnomedicinal manner and to increase the tone of the uterus after miscarriage or birth. For asthma, an infusion of the root bark has been used a use also common with traditional India's herbal practises. ${ }^{2}$

Its root is used in Ayurvedic medicine as an anti-inflammatory treatment for Swellings, cancers, rheumatism and scrofula, and in anxiety neurosis, as a sedative and hypnotic. The leaf has anti-inflammatory properties, Hepato-protective properties of antibacterial. Fruits and seeds have diuretic properties. The berries are used in cheese making as a substitute for rennet, to coagulate milk. Studies have shown that the activity of the extract of Withania was roughly equal to that of the extract of Panax ginseng. However, Withania somnifera has an advantage over Panax ginseng in that it does not seem to contribute to ginseng abuse syndrome, a disease of High blood pressure, water retention, muscle pain, and insomnia are characterised. ${ }^{3}$ Huge numbers of experimental and clinical studies performed on Ashwagandha are screened on different biological systems for their protection and effectiveness, but their information is scattered. Many review papers are also constructed, but most of them are only based on pharmacological and pharmacological (in vivo or in vivo) In vitro) profile profiles.

Table 1: Indication described in Ayurvedic Medicine ${ }^{4}$ -

\begin{tabular}{|l|l|}
\hline Murchha (syncope) & Apasmara (epilepsy) \\
\hline Shosha(cachexia) & Unmada (mania/psychosis) \\
\hline Karshya (emaciation) & Arsha (piles) \\
\hline Pramehapidika (diabetic carbuncle) & Arbuda (tumour) \\
\hline Gandamala (cervical lymphadenitis) & Bhagandara (fistula-in-ano) \\
\hline Guhya Vrana (ulcer in genitalia) & Vatarakta (gout) \\
\hline Kushtha (diseases of the skin) & Kilasa (vitiligo) \\
\hline Yonidosha (disorders of female genital tract) & Katigraha (stiffness in lumbosacral region) \\
\hline Gridhrasi (sciatica) & Hanugraha (lockjaw) \\
\hline Janustabdhata (stiffness of the knee) & Hrudgraha (cardiac failure) \\
\hline Asthibhanga (bone fracture) & Vidradhi (abscess) \\
\hline
\end{tabular}


Table 2: Ashwagandha Formulations ${ }^{5}$

\begin{tabular}{|l|l|}
\hline Shwagandhadi Churna & Ashwagandha Rasayana \\
\hline Ashwagandha Ghrita & Ashwagandharishta \\
\hline Ashwagandha Taila & Madhyamanarayana Taila \\
\hline Brihat Ashwagandha Ghrita & Brihachchhagaladyaghrita \\
\hline Saraswata Churna & Pramehamihira Taila \\
\hline Nagabala Ghrita & Madhusnuhi Rasayana \\
\hline
\end{tabular}

Table 3: Taxonomical classification ${ }^{6}-$

\begin{tabular}{l|}
\hline Kingdom- Plantae \\
\hline Superdivision- Spermatophyta \\
\hline Class- Mangnoliopsida- Dicotyledons \\
\hline Order- Tubiorae/ Solanales \\
Genus- Withania
\end{tabular}

Subkingdom- Tracheobionta

Division- Angiosperma

Subclass- Ateridae

Family- Solanaceae

Species- Withania Somnifera (L.) Dunal

\section{Rasa Panchaka-}

Rasa- Katu, Tikta Rasa, Guna- Laghu, Virya- Ushna Virya, Vipaka- Madhura

Geographical Distribution-Widespread in semiarid environments from the Mediterranean coast to Indian.

\section{Description ${ }^{7,8}$.}

A woody shrub, growing from a long, tuber out taproot, stellateomentose, up to $2 \mathrm{~m}$ in height.

Leaf- Simple, $2-11 \mathrm{~cm}$ in length, $1.5-9.0 \mathrm{~cm}$ in width, exstipulate, $6-20 \mathrm{~mm}$ long petiole, elliptical to the ovate-lanceolate blade, acute or rounded apex, acute to long decurrent base, $8-10 \mathrm{~cm}$ long and alternate vegetative shoots, $3-8 \mathrm{~cm}$ long and opposite reproductive shoots, arranged in pairs of one wide and smaller leaf, whole or wavy margin.

Inflorescence- Axillary, 2-25 yellow green, shot-pedicellate flowers, umbellate cyme. Perfect, radially symmetrical, campanulate, calyx with 5 acute triangular lobes, corolla twice the length of the calyx, 7-8 mm long, with 5 spreading or reflexed lanceolate lobes, stamen 5, slightly exserted, alternating filaments with petal lobes, partially fused with the corolla, superior, glorous, stigma bifid.

Fruit- Berry, globose, 5-6 mm in diameter, orangered, closed in green, membranous, calyx-inflated, 2.5 $\mathrm{cm}$ in diameter, roughly, and 5 angled slightly.

Seeds- Many, discoid, $2.5 \mathrm{~mm}$ pale yellow in diameter.

\section{Dried Root-}

General appearance- Straight and unbranched, the thickness varying with age. The main roots bear fibre like secondary roots. The outer surface of the root is buff to grey, yellow with longitudinal wrinkled. The crown consists of 2-6 remains of the stem base. The base of the stem is green, variously thickened, cylindrical, and longitudinally wrinkled. The roots break with short uneven fracture.

\section{Organoleptic Properties-}

Odour- Characteristic, horse-like.

Taste - Sweetish, yet bitter and astringent and slightly mucilaginous.

Microscopic Characteristics- The transverse section shows an exfoliated or crushed narrow band of yellowish cork, a narrow cortex filled with starch grains, a cork cambium of 2-4 diffused rows of cells, a secondary cortex of around 24 layers of compact parenchymatous cells, phloem consisting of a sieve tube, a corresponding cell, secondary hard xylem, forming a closed vascular ring separated by multiserial medullary rays, a few xylems parenchyma cells.

Powdered material - Thin cork walled, lignified, cubic or elongated cells, often indistinct and collapsed, with yellowish-brown contents, 2-3 cells deep in smaller roots up to 16 in larger primary roots, dusty white or grey to yellow-brown. Cortex parenchyma is composed of large thin-walled cells filled with starch granules, and sometimes containing calcium oxalate microsphenoidal crystals, Xylem elements are either 
tracheidal with bordered pits or more rarely, thickened reticulate vessels, thickened lignified walls and simple pits of xylem fibre. Abundant, simple or 2-4 compound starch, with a labelled, irregularly shaped hilum.

Cultivation and Economics- It was estimated that about 2000 tons of Ashwagandha roots were produced annually in India, but the requirement was estimated at 7000 tonnes. Because of its popularity due to largescale unrestricted exploitation, the need for Withania somnifera has increased sharply. As a result of the depletion of this medically important plant species from its natural habitat, it is now included in the International Union for the Conservation of Nature and Natural Resources list of threatened species. ${ }^{9}$

Withania Somnifera grows well with good drainage at pH 8.0 in sandy loam or light red soil. It can be cultivated at altitudes of between $600-1200 \mathrm{~m}$. The semitropical regions receiving rainfall of $500-750 \mathrm{~mm}$ are ideal for the cultivation of this rainy crop. During its growth period, the crop needs a dry season. The temperature that is most suitable for cultivation is between $20^{\circ} \mathrm{C}$ and $35^{\circ} \mathrm{c}$. Late winter rains are useful for the proper growth of the plant roots. ${ }^{10}$

Phyto-chemistry- Given the medicinal significance and the wide application of Withania Somnifera as a therapeutic agent, the apparent potential of Withania Somnifera as a positive health promoter in the global market is well established, which in turn has attracted the interest of various phytochemist's. Consequently, the chemistry of Withania Somnifera has been extensively studied, leading to the isolation and characterization of many classes of biologically and pharmacologically important chemical constituents. Alkaloids, steroidal compounds and ergostane and steroidal lactones are the biologically active chemical constituents (withaferin A, withanolides A-Y, withanone etc). Saponins containing additional acyl groups (sitoindosides IX and X) and withanolide glycosides called withanosides I to VII are additional constituents. ${ }^{11,12,13,14,15,16,17}$ More than 12 alkaloids, 40 withanolides and several sitoindosides have currently been isolated and reported from aerial components, roots, and berries of the Withania species (Mirjalili et al., 2009). In addition, several other secondary metabolites are borne by the plant, including flavonol glycosides, sterols, phenolics, chemical components such as withaniol, acylsteryl glucosides, starch, sugar reduction, hantreacotane, ducitol, a variety of amino acids including aspartic acid, proline tyrosine, alanine, glycine, glutamic acid, cystine, tryptophan and high iron content. ${ }^{18}$

Alkaloids- The medicinal properties of the root are due to many alkaloids being present. Eight brown alkaloids were identified in primary studies, the main alkaloid being "withanine" which showed sedative and hypnotic action. ${ }^{19,20}$

Later, the presence of several biochemically heterogeneous alkaloids was confirmed by chromatographic analysis of the root extract. Thirteen positive Dragendroff alkaloids were obtained from the Indian variety. Cuscohygrine; dlisopelletierine; anahygrine; choline; soniferine; withanine; anaferine; isopelletierine; tropine; pseudotropine; 3 alpha-tigloyloxtropine; 3 alpha-tropyltigloate; hygrine; mesoanaferine; withananine; hentriacontane; visamine; pyrazole derivative withasomnine; pseudowithanine and ashwagandine are the alkaloids identified. ${ }^{21,22,23}$ However the alkaloids of Withania Somnifera were not individually found to have any unique biological or pharmacological actions. The total alkaloid content of Indian chemotype roots ranges between $0.13 \%$ to $0.31 \%$. ${ }^{24,25}$ Withanolides- The chemical constituents of Withania Somnifera, especially noticeable in the leaves, are marked by a great deal of variability. This variability is marked by the inclusion of the withanolide group of differently substituted steroidal lactones. A group of compounds characterised by a C28 basic steroidal skeleton with a side chain of nine carbon atoms in which C-22 and C-26 are properly oxidised to form a six-membered $\delta$-lactone ring was given the name 'Withanolide.' A 22-hydroxyergostan-26-oic acid-26, 22-lactone can be described as the withanolide skeleton. ${ }^{26,27}$

As a general function of the molecule, a 1-keto-to-2system, located in ring A, was observed. From the biogenetic point of view, withanolides can be regarded as having a structure of the cholestane form with an 
extra methyl group at C-24 and with different oxygenated or double bonds located at different skeleton sites. $^{28}$

There are several novel structure variants of withanolides with either carbocyclic skeleton or side chain modifications and these have also been identified as withanolides-related modified withanolides or egostane-type steroids. These compounds are usually polyoxygenated, and an enzyme mechanism capable of oxidising all carbon atoms in a steroid nucleus is assumed to be in the plant that produces them. The characteristic feature in the $\mathrm{C} 8$ or $\mathrm{C} 9$-side chain of withanolides and ergosane-type steroids with a lactone or lacteal ring but the lactone ring can be either sixmembered or five-membered and can be fused through a carbon-carbon bond or an oxygen bridge with the carbocyclic portion of the molecule. Appropriate oxygen substituents can lead to bond splitting, new bondforming, ring aromatization and many other forms of rearrangements, resulting in compounds with new structures. Around 40 steroidal lactones from different parts of Withania Somnifera have been identified. Four unusual withanolides (16-en-27-deoxywithaferin-A; 2,3-dihydro-3b-hydroxy withanone; 27-acetoxy-3oxo-witha-1,4,24-trienolide; 2,3-dihydroxy-3 $\beta$-O-sulfate) were isolated and characterised by Misra et al. (2005) along with six known withanolides (24,25-dihydroxythanolide A; withanolide A; withanone; withaferin A; 27-hydroxywithanone; and 17-hydroxy withaferin A from Withania Somaferin A leaves. Further research has led to the isolation of two new and seven known root withanolides and five new Withanolide from Withania somnifera stem bark, namely withasomnilide, withasomniferanolide, somniferanolide, somniferawithanolide and somniwithanolide. 29,30

Withanolide biogenesis appears to be highly restricted to a few genera and the largest number of withanolides are developed by Withania Somnifera in diversified functional groups and segio/stereo forms, some of which have unique therapeutic significance Withanolides are subdivided according to their structural characteristics as a) un-substituted compounds at Withanolide E. Development of Withanolide differs from plant to plant. In (1965), Lavie and his associates described five well-defined chemotypes of this plant that grow in various locations in Israel, India and South Africa, i.e. morphologically similar, but differing in their leaf withanolide material. ${ }^{31}$

In Indian varieties of Withania Somnifera, described two chemo-types. The main Indian chemotype-I steroidal lactone is withanone and Withaferin $\mathrm{A}$, both of which contain 5 alpha-hydroxy- 6 alpha, 7 alpha-epoxy grouping the ability of different chemotypes to produce withanolides with different patterns of nuclear replacement is genetically regulated and withanolides are produced either with new nuclear replacement depending on the genotype of the parents or entirely new withanolides not present in the parents. Some of the plant's important withanolides are withaferin A, withanone, withanolide D and withanolide A. Two primary withanolides have been linked to much of the pharmacological function of Withania somnifera: withaferin A and withanolide D. These secondary triterpenoid ancestry metabolites occur mainly in Withania Somnifera leaves and roots, but with major variations in their ratio for Ex. Withaferin $\mathrm{A}$ is found primarily in leaves, while withanolide $\mathrm{D}$ is found primarily in roots. Withanolides have mostly been found in members of the solanaceae plant family i.e. Datura, Deprea, Dunalis, Iochroma, Discopodium, Trechonaetes, Physalis, Salpichroa, Withania, Witheringia, Jaborosa, Lycium, Nicandra, Tubocapsicum, Acnistus. Other species of this genus, besides Withania somnifera, include W. coagulans, $\mathrm{W}$. aristata and $\mathrm{W}$. frutescence are known to produce withanolides. ${ }^{32,33}$

Withaferin A- One of the main withanodal active principles extracted from the plant to which the curative properties of the leaves are related is withaferin $\mathrm{A}$, chemically characterized as $(4 \beta, 5 \beta, 6 \beta, 22 \mathrm{R})-5,6$ Epoxy-4,22,27-trihydroxy-1-oxoergosta-2,24-dien26-oic acid $\delta$-lactone. Remarkable bacteristic, antitumor, anti-arthritic and anti-inflammatory activities are confirmed to have been reported. ${ }^{34}$ Due to its ability to arrest dividing cells at metaphase, Withaferin A serves as a cancer inhibitor. Inhibitory growth activity against a variety of cancerous cell lines such as KB, Sarcoma 180 and carcinoma of Ehrlich ascites has 
been shown It also has radio-sensitizing effects on tumours and cancer. Recently, due to the existence of unsaturated lactone rings, this highly oxygenated steroidal lactone has been reported to have potent antiangiogenic activity. Opening the lactone ring or saturating the double bond by alkaline hydrolysis causes the loss of activity. ${ }^{35}$ It inhibits cyclooxygenase-2 (COX-2) but not cyclooxygenase-1 (COX-1), which is necessary for a chemotherapeutic/non-ulcerative antiinflammatory drug. Immunosuppressive activity on Blymphocyte proliferation has also been noted. Withaferin A yields 0.18 per cent in Indian plants on an airdry basis, while South African varieties produce 0.86 per cent of the compound. Performed TLC densitometry quantitative analysis of Indian chemotypes of Withania Somnifera and found that withaferin-A was completely absent in the roots, stems, seeds and persistent calyx of intact plant fruits, except in leaves (1.6 per cent). Withaferin A was also observed in roots later with advanced analytical methods, but its concentration in roots was much less compared to leaves. ${ }^{36,37}$

\section{Classical applications of Ashwagandha (Withania} Somnifera) ${ }^{38,39}$ - Ashwagandha receives the unique name as root smells like horse (Ashwa) and it is believed that it provides the strength of a horse on guzzling. Different parts of Ashwagandha have significant therapeutic effects, either as a whole plant extract or as separate constituents. When fresh powder from the plant is used the best outcomes occur.

Ashwagandha root is very useful for its effective narcotic, diuretic, tonic, aphrodisiac, anthelmintic, astringent, tumour antiangiogenic, antimutagenic, thermogenic and stimulant properties. Roots are equally effective against constipation, weakness, goitre, rheumatoid, vitiated leucoderma conditions, insomnia and nervous disorders, lead-induced DNA damage, etc.

Ashwagandha root paste effectively reduces the occurrence of asthma, arthritis, rheumatoid arthritis, osteoarthritis, carbuncles, ulcers, leucoorrhea, boils, pimples, flatulent colic, piles, and painful swelling when applied locally as an anti-arthritic agent.
Antioxidant property ${ }^{40,41}$ - Several studies have shown that Ashwagandha can be used as a natural source of healthy antioxidant agents. Withania Somnifera acts as a potent antioxidant and increases the levels of three natural antioxidant enzymes such as superoxide dismutase, catalase, and glutathione peroxidase. Likewise, oral administration of Withania Somnifera. A rise in lipid peroxidation in mice and rabbits was prevented by Somnifera extract. The activity of Withnaia in antioxidants. Somnifera has been shown in mice and withanolides, glycowithanolides and VII$\mathrm{XX}$ sitoindosides were suggested to be imparted. In people treated with Withania, a substantial increase in haemoglobin, red blood cell count, hair melanin and reduced serum cholesterol was observed.

Since they are rich in lipids and iron, the brain and nervous system are comparatively more vulnerable to free radical damage than other tissues, both known to encourage the generation of reactive oxygen species. In cerebral ischemia, free radical nervous tissue damage can be responsible for neuronal loss and may be implicated in ageing and neurodegenerative disorders, such as epilepsy, schizophrenia, Parkinson's, Alzheimer's and other diseases. The active tenets of Withania increase in levels of endogenous superoxide dismutase (SOD), catalase (CAT), glutathione peroxidase (GPX) and ascorbic acid are recorded for somnifera, sitoindosides VII-X and withaferin A (glycowith-anolides), with a concomitant decrease in lipid peroxidation. The active principle of Withania Somnifera a root has antioxidant effects like Anti-stress, cognition facilitating, anti-inflammatory and anti-ageing effects.

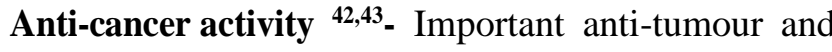
radio-sensitizing withanolides are stated to be withaferin $\mathrm{A}$ and withanolide $\mathrm{D}$. Another constituent of $\mathrm{W}$.

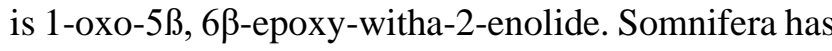
been reported to minimise UV-induced skin carcinoma. Withaferin A functions as a mitotic poison that arrests the metaphase division of the cultured cells of human larynx carcinoma. A major dose-dependent delay in the growth of Ehrlich ascites carcinoma, sarcoma 180, and sarcoma Black and E 0771 mammary adenocarcinoma has also occurred. Methanolic extract of Withania Somnifera has been used to proliferate 
stem cells. It also decreases breast, lung, central nervous system and colon cancer cell lines by reducing their viability in twelve dependent ways and therefore holds promise as a chemotherapeutic agent. Withaferin A-mediated breast cancer cell viability suppression correlated with the induction of apoptosis characterized by DNA condensation, DNA fragmentation associated with cytoplasmic histone, and poly (ADP-ribose)-polymerase cleavage. Chemo-preventive activity is due in part to the extract's antioxidant/free radical scavenging activity. Alteration of the cytoskeleton architecture by covalently binding an nexin II, anti-tumour potential by inhibition of pro-theasomal chymotrypsin-like activity and induction of apoptosis by inhibition of protein kinase $\mathrm{C}$ or activation of caspase- 3 has also been investigated. These findings indicate anti-tumour activity as well as enhancing the effects of radiation.

Anti-inflammatory properties $^{44,45}$ - Ashwagandha works by supplement inhibition, lymphocyte proliferation, and delayed type hypersensitivity as an anti-inflammatory agent. In several rheumatological disorders, Withania Somnifera extracts have shown anti-inflammatory effects. The extract was found to reduce the content of glycosaminoglycans in granuloma tissue by almost $100 \%$ and to decouple oxidative phosphorylation by significantly reducing the ADP/O ratio in granuloma tissue mitochondria and increasing the activity of the $\mathrm{Mg} 2+$ dependent-ATPase enzyme and subsequently reducing the activity of succinate dehydrogenase in granuloma tissue mitochondria. Studies indicate that inhibition of cyclooxygenase could be involved in the mechanism of action of Withania Somnifera.

Anti-microbial activities ${ }^{46}$ - For the first time, the antibacterial properties of this multipronged medicinal plant were documented against Salmonella aurens. Antimicrobial activity against a variety of bacteria and fungi assigned to withanolide has been documented over the past decade. However, current literature indicates that to explore its potential in the treatment of other infectious diseases as well this herb should be studied more extensively.
Anti-arthritic properties ${ }^{47}$ - In acute rheumatoid arthritis, Ashwagandha powder has been found useful and reduces the pain associated with arthritis. The active ingredient withaferin A was attributed to this property.

Anti-stress and Aphrodisiac activity ${ }^{48-}$ Bhattacharya has documented anti-stress activity associated with glycosides (sitoindosides VII and VIII) present in this plant (1987; $2000 \&$ 2003). (1987; $2000 \& 2003)$. The research conducted supported the utility of Ashwagandha as an adaptogen against antistress. In the treatment of spermatopathy, impotence and seminal depletion, Ashwagandha is also used as a tonic and men who used the herb enjoyed higher vigour output. The higher concentrations in the roots of this plant of in-organic elements such as $\mathrm{Fe}, \mathrm{Mg}, \mathrm{K}$ and $\mathrm{Ni}$ play an important role in the drug's diuretic and aphrodisiac function. For the healing of sterility in women, root decoction boiled with milk and ghee is recommended.

Effect on cardiovascular system ${ }^{49}$ - Assessment of the hypoglycemic as well as diuretic and hypo-cholesterolemic effects of Ashwagandha root in humans revealed that the treatment of subjects with type 2 diabetes and mildly hypercholesterolemic can be initiated with a powder extract for 30 days, resulting in a reduced in blood glucose level comparable to that of oral hypoglycemic medication. Significant increases have also been observed in serum cholesterol and triglycerides and low-density lipoproteins.

Cardiovascular Protection ${ }^{50}$ - The Withania Somnifera extracts are associated with hypotensive effects due to autonomic ganglion blocking action as well as a depressant action on the higher cerebral centres. Withania Somnifera has recently been verified as a cardioprotective agent, offering a scientific basis for the use of this medicinal plant as Maharasayana in Ayurveda. Effect on nervous system ${ }^{\mathbf{5 1 , 5 2}}$ - Ashwagandha is said to have the central nervous system sedative rather than stimulatory action, making it a superior nervous irritability drug in fatigue. The concentration of neurotransmitters, believed to play an important role in brain functions such as memory, is altered by Ashwagandha. The effects of Ashwagandholine are correlated with 
the nervous system (root extracts). It encourages barbiturate, ethanol and urethane-induced hypnosis in mice and has caused relaxant and antispasmodic effects in the intestinal, uterine, tracheal and vascular muscles against various agents that create smooth muscle contractions. The bioactive compounds are reported to affect the events in the cholinergic-signal transduction cascade of the cortical and basal forebrain preferentially. The effects of Withania Somnifera extracts on cognition and memory can be partially explained by the drug-induced improvement of the capacity of the cortical muscarinic acetylcholine receptor. Ashwagandha has been historically used as a tonic and no tropic agent in general. Improvements in scopolamine-induced memory deficits in mice have also been associated with Withania Somnifera extracts, by inhibiting haloperidol or reserpine-induced catalepsy attributed to potent antioxidants, antiperoxidant and free radical quenching properties, also display an antiparkinsonian effect on neuroleptic-induced catalepsy.

Effect on Immunity ${ }^{\mathbf{5 3}}$ - Withania Somnifera is known to have Immuno-potentiating and myelo-protective effects by increasing interferon (IFN)- $\gamma$, interleukin (IL)-2 and granulocyte-macrophage colony-stimulating factor levels in normal and cyclophosphamidetreated mice. As the plant is iron-rich, it adds to the number of red blood cells. The Withania Somnifera is more complex than suppressing the immune/inflammatory response in the immune system. The active compound withanolide $\mathrm{A}$ in the roots of Withania Somnifera greatly increases the T-helper 1 (Th1) cytokine expression levels, as well as the CD4 and CD8 counts. It also increases the dose-dependent function of natural killer (NK) cells with a quicker recovery of CD4+ T cells in immune-suppressed animals.

Immunomodulatory properties ${ }^{54}$ - For their immunomodulatory and central nervous system effects, glycowithanolides and a mixture of IX and X sitoindosides isolated from Withania Somnifera were evaluated. Both compounds, administered orally (50-200 $\mathrm{mg} / \mathrm{kg}$ orally), also developed significant anti-stress activity in albino mice and rats. In both young and old rats, they also improved understanding, acquisition, and memory retention. Withania Somnifera root extract was tested in three myelosuppression models in mice for immunomodulatory effects: cyclophosphamide, azathioprine, or prednisolone. ${ }^{143}$ Compared to controls, substantial increases in haemoglobin concentration, red blood cell count, white blood cell count, platelet count and body weight were observed in mice treated with Withania Somnifera. There have also been studies of a large increase in hemolytic antibody responses to human erythrocytes (indicating immunestimulatory activity).

The rejuvenating effect of Ashwagandha ${ }^{55,56}$ - Withania Somnifera has been reported to have a growthpromoting effect when administered in powdered form alone or in conjunction with other medicines. Withanolides are linked to growth-promoting activity. A significant improvement in haemoglobin, packed cell volume, mean corpuscular volume, serum iron, body weight, handgrip and total proteins were reported in the study conducted in both children and elderly people. In adults, serum cholesterol was reduced, and nail calcium was maintained. The sedimentation rate of erythrocytes decreased dramatically and $71.4 \%$ of them showed increased vigour. In short, these studies indicate that Withania Somnifera as a general health tonic can prove useful in younger as well as older populations.

In humans, the hypoglycemic and diuretic effects of Ashwagandha roots were also assessed. A blood glucose decrease similar to that of an oral hypoglycemic drug has been observed. There were also significant increases in urinary sodium, urine volume and serum cholesterol, triglycerides, and low-density lipoproteins.

\section{DISCUSSION AND CONCLUSION}

The available scientific data support the conclusion that Ashwagandha (WS) is a real potent Rasayana (regenerative tonic). The literature revealed that Ashwagandha (WS) is a rich source of pharmacologically and medicinally important compounds such as withaferins, sitoindosides, and a variety of beneficial alkaloids. Ashwagandha is used as a household remedy by Indians, who consider it as best tonic for old person and 
children and as Vrishya (aphrodisiac) by young people. It is one the most using nervine tonics of Ayurveda. The Ashwagandha has also been widely studied for their various pharmacological activities like an Antioxidant, adaptogen, memory enhancing, anti-parkinsonian, antivenom, antiinflammatory, antitumor properties. Various other effects like immunomodulation, hypolipidemic, antibacterial, cardiovascular protection, sexual behaviour has also been studied. Ashwagandha (WS) used as a multi-purpose medicinal herb in Ayurvedic medicine for centuries, a more clinical trial should be conducted to support its therapeutic use.

\section{REFERENCES}

1. Ven Murthy MR, K Ranjekar P, Ramasamy C, Deshpande M. Scientific basis for the use of Indian ayurvedic medicinal plants in the treatment of neurodegenerative disorders: 1. Ashwagandha. Central Nervous System Agents in Medicinal Chemistry (Formerly Current Medicinal Chemistry-Central Nervous System Agents). 2010 Sep 1;10(3):238-46.

2. Sangwan RS, Chaurasiya ND, Misra LN, Lal P, Uniyal GC, Sharma R, Sangwan NS, Suri KA, Qazi GN, Tuli R. Phytochemical variability in commercial herbal products and preparations of Withania somnifera (Ashwagandha). Current Science. 2004 Feb 10:461-5.

3. K. Nishteswar, K. Hemadri (First edition), Dravyaguna Vijnana, Chaukhambha Samskrit Pratishthana, 2013.121.

4. Bone K. Clinical applications of Ayurvedic and Chinese herbs. Monographs for the Western Herbal Practitioner. Australia: Phytotherapy. 1996:137-41.

5. Anonymous, The Ayurvedic Pharmacopeia of India, part I and I vol I, Dept. of Ayush, Ministry of health \& family welfare, New Delhi., 2000.

6. Anonymous, The Ayurvedic Formulary of India, Part 1, Dept. of Ayush, Ministry of health \& family welfare, New Delhi., 2004.

7. Ibidem (11) and (12) The API and The AFI, 2003.

8. Anonymous, Database on Indian medicinal plants CCRAS, New Delhi vol-3. 88.

9. Anonymous, Database on Indian medicinal plants CCRAS, New Delhi vol-3. 88.

10. Tiwari KN, Sharma NC, Tiwari V, Singh BD. Micropropagation of Centella Asiatica (L.), a valuable medicinal herb. Plant Cell, Tissue and Organ Culture. 2000 Dec;63(3):179-85.
11. Sandhya S, Sushil K. Withania somnifera: the Indian ginseng ashwagandha. Withania somnifera: the Indian ginseng ashwagandha. 1998.

12. Mishra LC, Singh BB, Dagenais S. Scientific basis for the therapeutic use of Withania somnifera (ashwagandha): a review. Alternative medicine review. 2000 Aug $1 ; 5(4): 334-46$

13. Gupta GL, Rana AC. PHCOG MAG.: Plant review Withania somnifera (Ashwagandha): A review. Pharmacognosy Reviews. 2007 Jan;1(1):129-36.

14. Baraiya BR, Tiwari G, Sonakia VK. Alkaloid concentration in different parts of growing crop of ashwagandha (Withania somnifera) at different growth intervals. J. Med. Arom. Plant Sci. 2005;27:439-42.

15. Matsuda H, Murakami T, Kishi A, Yoshikawa M. Structures of withanosides I, II, III, IV, V, VI, and VII, new withanolide glycosides, from the roots of Indian Withania somnifera DUNAL. and inhibitory activity for tachyphylaxis to clonidine in isolated guinea-pig ileum. Bioorganic \& medicinal chemistry. 2001 Jun 1;9(6):1499-507.

16. El Moussaoui A, Bourhia M, Jawhari FZ, Mechchate H, Slighoua M, Bari A, Ullah R, Mahmood HM, Ali SS, Ibenmoussa S, Bousta D. Phytochemical Identification, Acute, and Sub-Acute Oral Toxicity Studies of the $\mathrm{F}$

17. Sangwan RS, Chaurasiya ND, Lal P, Misra L, Tuli R, Sangwan NS. Withanolide A is inherent de novo biosynthesized in the roots of the medicinal plant Ashwagandha (Withania somnifera). Physiologia plantarum. 2008 Jun;133(2):278-87.

18. Misra L, Mishra P, Pandey A, Sangwan RS, Sangwan NS, Tuli R. Withanolides from Withania somnifera roots. Phytochemistry. 2008 Feb 1;69(4):1000-4.

19. Gupta GL, Rana AC. PHCOG MAG.: Plant review Withania somnifera (Ashwagandha): A review. Pharmacognosy Reviews. 2007 Jan;1(1):129-36.

20. Majumdar DN, Guha PC. Indian medicinal plants. Part I. Withania somnifera. Journal of the Indian Institute of Science. 1933;16:29.

21. Majumdar RC. Ancient Indian Colonization in Southeast Asia. Oriental Institute; 1955.

22. Gupta GL, Rana AC. PHCOG MAG.: Plant review Withania somnifera (Ashwagandha): A review. Pharmacognosy Reviews. 2007 Jan;1(1):129-36.

23. Sandhya S, Sushil K. Withania somnifera: the Indian ginseng ashwagandha. Withania somnifera: the Indian ginseng ashwagandha.. 1998. 
24. Nigam KB, Kandalkar VS. Ashwagandha - Advances in Horticulture, Vol. 11. Medicinal and Aromatic plants. Malhorta Publishing House, New Delhi, India. 1995; 337-344

25. Bargale SS, Tripathy TB, Shashirekha HK. Phyto Physicochemical Profile of Withania somnifera Dunal (Solanaceae). Journal of Drug Delivery and Therapeutics. 2019 Jun 15;9(3-s):263-8.

26. Majumdar DN, Guha PC. Indian medicinal plants. Part I. Withania somnifera. Journal of the Indian Institute of Science. 1933;16:29.

27. Tursunova RN, Maslennikova VA, Abubakirov NK. Withanolides in the vegetable kingdom. Chemistry of Natural Compounds. 1977 Mar;13(2):131-8.

28. Mirjalili MH, Moyano E, Bonfill M, Cusido RM, Palazón J. Steroidal lactones from Withania somnifera, an ancient plant for novel medicine. Molecules. 2009 Jul;14(7):2373-93.

29. Kirson I, Glotter E, Abraham A, Lavie D. Constituents of Withania somnifera dun-XI: the structure of three new withanolides. Tetrahedron. 1970 Jan 1;26(9):2209-19.

30. Tursunova RN, Maslennikova VA, Abubakirov NK. Withanolides in the vegetable kingdom. Chemistry of Natural Compounds. 1977 Mar;13(2):131-8.

31. Roja G, Heble MR, Sipahimalani AT. Tissue cultures of Withania somnifera: morphogenesis and withanolide synthesis. Phytotherapy Research. 1991 Aug;5(4):1857.

32. Lavie D, Glotter E, Shvo Y. 1371. Constituents of Withania somnifera Dun. Part IV. The structure of withaferin A. Journal of the Chemical Society (Resumed). 1965:7517-31.

33. Ray S, Jha S. Production of withaferin A in shoot cultures of Withania somnifera. Planta Medica. 2001;67(05):432-6.

34. Tursunova RN, Maslennikova VA, Abubakirov NK. Withanolides in the vegetable kingdom. Chemistry of Natural Compounds. 1977 Mar;13(2):131-8.

35. Devi PU, Sharada AC, Solomon FE, Kamath MS. In vivo growth inhibitory effect of Withania somnifera (Ashwagandha) on a transplantable mouse tumour, Sarcoma 180. Indian journal of experimental biology. 1992 Mar 1;30(3):169-72.

36. Yoshida M, Hoshi A, Kuretani K, Ishiguro M, Ikekawa N. Relationship between chemical structure and antitumor activity of withaferin A analogues. Journal of Pharmacobio-Dynamics. 1979;2(2):92-7.
37. Sharma ML, Srivastava P, Sharma P. Biochemistry and pharmacological use of withania somnifera (ashwagandha): a medicinal plant. International Journal of Pharmacology \& Biological Sciences. 2013 Aug 1;7(2).

38. Kulkarni RR, Patki PS, Jog VP, Gandage SG, Patwardhan B. Treatment of osteoarthritis with a herbomineral formulation: a double-blind, placebocontrolled, cross-over study. Journal of ethnopharmacology. 1991 May 1;33(1-2):91-5.

39. Singh N, Bhalla M, de Jager P, Gilca M. An overview on ashwagandha: a Rasayana (rejuvenator) of Ayurveda. African Journal of Traditional, Complementary and Alternative Medicines. 2011;8(5S).

40. Bhattacharya SK, Satyan KS, Ghosal S. Antioxidant activity of glycowithanolides from Withania somnifera. Indian journal of experimental biology. 1997 Mar 1;35:236-9.

41. Dhuley JN. Therapeutic efficacy of Ashwagandha against experimental aspergillosis in mice. Immunopharmacology and immunotoxicology. 1998 Jan 1;20(1):191-8.

42. Mathur S, Kaur P, Sharma M, Katyal A, Singh B, Tiwari M, Chandra $\mathrm{R}$. The treatment of skin carcinoma, induced by UV B radiation, using 1-oxo- $5 \beta, 6 \beta$-epoxywitha-2-enolide, isolated from the roots of Withania somnifera, in a rat model. Phytomedicine. $2004 \mathrm{Jul}$ 20;11(5):452-60.

43. Kuttan G. Use of Withania somnifera Dunal as an adjuvant during radiation therapy. Indian journal of experimental biology. 1996 Sep 1;34(9):854-6.

44. Rasool M, Varalakshmi P. Immunomodulatory role of Withania somnifera root powder on experimental induced inflammation: An in vivo and in vitro study. Vascular pharmacology. 2006 Jun 1;44(6):406-10.

45. Anbalagan K, Sadique J. Role of prostaglandins in acute phase proteins in inflammation. Biochemical medicine. 1984 Apr 1;31(2):236-45.

46. Owais M, Sharad KS, Shehbaz A, Saleemuddin M. Antibacterial efficacy of Withania somnifera (ashwagandha) an indigenous medicinal plant against experimental murine salmonellosis. Phytomedicine. 2005 Mar 22;12(3):229-35.

47. Bector NP, Puri AS, Sharma D. Role of Withania somnifera (Ashwagandha) in various types of arthropathies. Ind Jour Med Res. 1968 Oct;56:1581-3.

48. Singh N, Bhalla M, de Jager P, Gilca M. An overview on ashwagandha: a Rasayana (rejuvenator) of Ayurveda. African Journal of Traditional, Complementary and Alternative Medicines. 2011;8(5S). 
49. Ojha SK, Arya DS. Withania somnifera Dunal (Ashwagandha): a promising remedy for cardiovascular diseases. World J Med Sci. 2009;4(2):156-8.

50. Sukumar BS, Tripathy TB, Shashirekha HK, Shetty SK. Ef icacy of Ashwagandha (withania somnifera [1.] Dunal) in improving cardiorespiratory endurance (VO2 max test) in healthy subjects.

51. Choudhary D, Bhattacharyya S, Bose S. Efficacy and safety of Ashwagandha (Withania somnifera (L.) Dunal) root extract in improving memory and cognitive functions. Journal of dietary supplements. 2017 Nov 2;14(6):599-612.

52. Sehgal N, Gupta A, Valli RK, Joshi SD, Mills JT, Hamel E, Khanna P, Jain SC, Thakur SS, Ravindranath V. Withania somnifera reverses Alzheimer's disease pathology by enhancing low-density lipoprotein receptorrelated protein in the liver. Proceedings of the National Academy of Sciences. 2012 Feb 28;109(9):3510-5.

53. Davis L, Kuttan G. Effect of Withania somnifera on cytokine production in nol and cyclophosphamide treated mice. Immunopharmacology and immunotoxicology. 1999 Jan 1;21(4):695-703.

54. Ghosal S, Lal J, Srivastava R, Bhattacharya SK, Upadhyay SN, Jaiswal AK, Chattopadhyay U. Immunomodulatory and CNS effects of sitoindosides IX and X, two new glycowithanolides from Withania somnifera. Phytotherapy Research. 1989;3(5):201-6.

55. Andallu B, Radhika B. Hypoglycemic, diuretic and hypocholesterolemic effect of winter cherry (Withania somnifera, Dunal) root.

56. Krutika J, Tavhare S, Panara K, Kumar P, Karra N. Studies of Ashwagandha (Withania somnifera Dunal). Int J Pharm Biol Arch. 2016;7(1):1-1.

\section{Source of Support: Nil Conflict of Interest: None Declared}

How to cite this URL: Bargale Sushant Sukumar: Miracle Ayurvedic Herb - Ashwagandha (Withania Somnifera Dunal). International Ayurvedic Medical Journal \{online\} 2021 \{cited August 2021\} Available from: http://www.iamj.in/posts/images/upload/1778_1788.pdf 